\title{
Characterization Phyto-Socio-Ecological of Agrostis capillaris L. Grasslands
}

\author{
Mirela CIREBEA, Ioan ROTAR, Roxana VIDICAN, Florin PĂCURAR, Anamaria MALINAS, Ovidiu RANTA \\ Faculty of Agriculture. University of Agricultural Sciences and Veterinary Medicine Cluj-Napoca, \\ Manăștur Street, 3-5, 400372, Romania \\ *corresponding author: ioan.rotar@usamvcluj.ro
}

Bulletin UASVM series Agriculture 73(2)/2016

Print ISSN 1843-5246; Electronic ISSN 1843-5386

DOI 10.15835/buasvmcn-agr: 12450

\begin{abstract}
Floristic composition of the pastures is a mirror action and practical factors applied station and management (maintenance and how to use). The purpose of this research is to assess the state of the biodiversity and pastoral value for Agrostis capillaries L. grasslands from the mountain area. Also, there has been analyzed the floristic composition and a series of ecological indexes, respectively humidity, soil reaction, temperature, and nitrogen. We examined how estimated species diversity patterns changed with varying survey intensity from Băișoara village, Cluj County, Romania, an Agrostis capillaries L. grasslands. The experiment was performed in 2015. The vegetation observations were made on 29 plots. In this descriptive plot been identified the type of grassland Agrostis capillaries, described in boreal at altitudes between 850 and 1200, generally starting on exhibitions east until the south $\left(90^{\circ}\right.$ - $180^{\circ}$ ).The floristic composition of the Agrostis capillaries grasslands from the studied area is influenced by the local conditions and the intensity of the exploitation, these surfaces being under-exploited.
\end{abstract}

Keywords: Băișoara village, ecology, grassland.

\section{INTRODUCTION}

In Baisoara village have been studied one grassland plots of 500 hectares. The purpose of this research is to assess the state of the biodiversity and pastoral value for Agrostis capillaries L. grasslands from the mountain area. Also, there has been analyzed the floristic composition and a series of ecological indexes, respectively humidity, soil reaction, temperature, and nitrogen. Other aspects taken into account were the agronomical and anthropogenic specters. The analyzed grasslands are placed in the perimeter of Băișoara village, from Cluj County.

The purpose of this research is to assess the state of the biodiversity and pastoral value for Agrostis capillaries L. grasslands from the mountain area. Also, there has been analyzed the floristic composition and a series of ecological indexes, respectively humidity, soil reaction, temperature and nitrogen. Other aspects taken into account were the agronomical and anthropogenic specters.

\section{MATERIAL AND METHODS}

In this study, we examined how estimated species diversity patterns changed with varying survey intensity from Băișoara village, Cluj County, Romania, on an Agrostis capillaries L. grasslands. The experiment was performed in 2015 (Fig. 1).

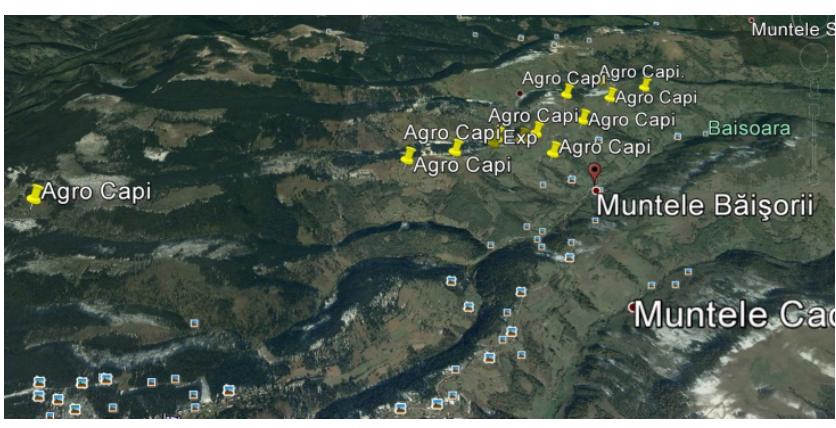

Fig. 1. Geographical location of the Baisoara village

The area shows a typical mountain climate, with altitudes between 850 and $1200 \mathrm{~m}$ above 
sea level. It is characterized by a high variation of land use and too climatic conditions in the area and a fine-grained mosaic of different land uses, including substantial amounts of semi-natural vegetation with $5.2^{\circ} \mathrm{C}$ average temperature (Păcurar et al, 2014). The vegetation observations were made on 29 plots.

Data analysis. The floristic composition was interpreted using an improved Braun-Blanquet scale with subdivisions (Pacurar and Rotar, 2014). Requirements plants to environmental factors (temperature, soil moisture and soil reaction) have been materialized by values of indicator species (from 1-9) after Ellenberg $(1952,1992)$ updated (Pacurar and Rotar, 2014). Plant resistance to mechanical interference, such as mowing, grazing and crushed materialized by value indicator (from 1-9) after (Dierschke et al., 2002), and the names of appropriate species depending on the category disturbance were taken after Pacurar and Rotar (2014). Based on data from spectrum it can calculate the average indicator of a phytocoenosis. This may be unweighted or weighted. Assigning a phytocoenosis feed is achieved at the expense calculated weighted average indicator value. Using descriptive statistics (Cristea et al. 2004) analyzes were performed which are divided into two categories: central tendency parameters and indicators of scattering data. In the central tendency, parameters included those processes provide a representative value (central) measured for the data stream. There are three estimators that can be used for this purpose: the mean, median, and module (http://statisticasociala.tripod. com/parametri.htm).

\section{RESULTS AND DISCUSSIONS}

In this descriptive plot was identified Agrostis capillaries type of grassland,which belongs to the series Agrostis capillaries, of characteristic areas which moderately humid habitats, wet soils, mesobasic, moderately acid, sometimes skeletal or poor humus (Tucra et al., 1987).

In our case, Agrostis capillaris type, was described in boreal floor at altitudes between 850 and 1200, in general exhibitions starting from the eastern to the southern regions $\left(90^{\circ}-180^{\circ}\right)$ on land with a slope about 10 degrees mean sometimes reaching up to a maximum inclination of 32 degrees (Tab. 1).Of the total proportion of the 50 relevees this type was found in 29 locations, representing 58\% of grasslands studied. Agrostis capillaris type is exploited most often mixed (mowing and then grazing) as shown by the Garda in 2010. In this type, woody vegetation cover has an average of $5.75 \%$ (table 1), the amplitude data is high ( $\max =15 \%=2 \%$ minimum), and cover the most common being $2 \%$ (Module $=2 \%$ ). Stub presence in most cases absent entirely (module $=0 \%$; median $=0 \%$ ). The situation is similar in the case of rocks ( mean $=0 \%$, module $=0 \%$; median $=0 \%$ ) and stones (median $=0 \%$, module $=0 \%$; median $=0 \%$ ). Fallow molehills have a presence, in generally $1.75 \%$ (mean $=2.6 \%$; median $=1.5 \%)$. In this types is meet molehills without fallow, but with a small participation $0.8 \%$ (mean $=1 \%$; module $=1 \%$ ). Overall coverage herbaceous vegetation is averaging $79.79 \%$, data distribution is one relatively normal (obliquity $=-0.34 \%$ ) and platikurtic (vaulting = -0.62; Annex 1).

The Poaceae family makes its presence felt in herbaceous cover with a $42.03 \%$ average participation (Tab. 1), data having a high amplitude (minimum $=0.5 \%$ and maximum $=27.5 \%$ ). At Poaceae addition of the dominant species (Agrostis capillaries) with a large Adm, is present species Holcus lanatus with $8.26 \%$ and most frequent coverage value is $11.25 \%$, Adm followed by Anthoxanthum odoratum with $2.73 \%$ having the frequency of covering $2.75 \%$, and then Festuca rubra with an average of covering $1.29 \%$.

Regarding the distribution of data on only three species, this is normal and symmetric (obliquity $=-0.20 ;-1.87$ ), except the species Anthoxanthum odoratum, which has a negative asymmetry (obliquity $=2.57$ ) at Poaceae family other species with smaller presence. Among the species of the Poaceae family species are evident for this type of grassland following: Agrostis capillaries, Anthoxanthum odoratum and Cynosurus cristatus $(\mathrm{K}=\mathrm{V})$. Two common species are present (Briza media and Holcus lanatus, $\mathrm{K}=\mathrm{IV}$ ), and two indicator species Festuca rubra and Phleum pratense (K = III). Accompanying species are 2 , and the other five species are random $(\mathrm{K}=\mathrm{I})$.

Regarding the participation of Cyperaceae and Juncaceae families in this type of meadow is found that have averaged coverage of $1.5 \%$.

Fabaceae family participates in the floristic composition, with an average of $18.77 \%$, recording a maximum of $17.5 \%$ of the species Trifolium pratense. Data collected on their participation 
Tab. 1. Floristic composition of the type of grassland Agrostis capillaries and specific requirement of ecological, agronomic and anthropogenic (B - BioForm, T - temperature, U - humidity, R - soil reaction, $\mathrm{N}$ - nutrition, $\mathrm{C}$ - tolerance of mowing, $\mathrm{P}$ - tolerance of grazing, $\mathrm{S}$ - tolerance of crushed, $\mathrm{VF}$ - fodder value, $\mathrm{H}$ - hemerobie, UR - urbanophile, SO - sociological category)

\begin{tabular}{|c|c|c|c|c|c|c|c|c|c|c|c|c|c|c|}
\hline \multicolumn{5}{|c|}{ Ecological Indexes } & \multicolumn{4}{|c|}{$\begin{array}{l}\text { Agronomical } \\
\text { indexes }\end{array}$} & \multicolumn{3}{|c|}{$\begin{array}{l}\text { Anthropogenic } \\
\text { indexes }\end{array}$} & \multirow{2}{*}{$\begin{array}{l}\text { Stand conditions } \\
\text { Altitude }(m)\end{array}$} & \multicolumn{2}{|l|}{$\overline{\mathbf{x}}$} \\
\hline & & & & & & & & & & & & & & \\
\hline & & & & & & & & & & & & Slope $\left({ }^{\circ}\right)$ & 30.84 & \\
\hline & & & & & & & & & & & & Exposition & S, S-V & \\
\hline & & & & & & & & & & & & Land use & grazing & \\
\hline & & & & & & & & & & & & Grassland type & $\begin{array}{l}\text { Agrostis } \\
\text { capillaries }\end{array}$ & \\
\hline & & & & & & & & & & & & General cover (\%) & 79.79 & \\
\hline & & & & & & & & & & & & Wooden vegetation cover (\%) & 5.75 & \\
\hline & & & & & & & & & & & & Stubs (\%) & - & \\
\hline & & & & & & & & & & & & Rocks (\%) & - & \\
\hline & & & & & & & & & & & & Stones (\%) & - & \\
\hline & & & & & & & & & & & & Fallow molehills (\%) & 1.75 & \\
\hline & & & & & & & & & & & & Fresh molehills (\%) & 0.8 & \\
\hline & & & & & & & & & & & & Landslides (\%) & 6.75 & \\
\hline & & & & & & & & & & & & Erosion (\%) & 1 & \\
\hline & & & & & & & & & & & & Swamps (\%) & - & \\
\hline & & & & & & & & & & & & SPECIES & Adm & $\mathrm{K}$ \\
\hline B & $\mathrm{T}$ & $\mathbf{U}$ & $\mathbf{R}$ & $\mathbf{N}$ & C & $\mathbf{P}$ & $\mathbf{S}$ & VF & So & $\mathbf{H}$ & UR & POACEAE & 42.03 & \\
\hline HT & $\mathrm{x}$ & $\mathrm{x}$ & $\mathrm{x}$ & 4 & 6 & 5 & 5 & $\mathrm{n}$ & 6 & $2-4$ & 3 & Agrostis capillaris $L$. & 23.71 & $\mathrm{~V}$ \\
\hline HT & $\mathrm{x}$ & $\mathrm{x}$ & 5 & $\mathrm{x}$ & 7 & 5 & 5 & $\mathrm{n}$ & 4 & $2-4$ & 2 & $\begin{array}{l}\text { Anthoxanthum odoratum L. } \\
\text { s. str. }\end{array}$ & 2.73 & $\mathrm{~V}$ \\
\hline HT & $\mathrm{x}$ & $\mathrm{x}$ & $\mathrm{x}$ & 3 & 4 & 4 & 4 & $\mathrm{n}$ & 5 & $2-3$ & 2 & Briza media L. & 0.50 & IV \\
\hline $\mathrm{H}$ & $\mathrm{x}$ & 4 & 8 & 5 & 4 & 4 & 3 & $\mathrm{n}$ & 7 & $3-4$ & 3 & Bromus inermis Leyss. & 0.50 & I \\
\hline HT & 5 & 5 & $\mathrm{x}$ & 4 & 7 & 7 & 7 & $\mathrm{n}$ & 7 & $2-3$ & 2 & Cynosurus cristatus L. & 1.53 & $\mathrm{~V}$ \\
\hline HT & $\mathrm{x}$ & $\mathrm{x}$ & 2 & 3 & 3 & 4 & 4 & $\mathrm{n}$ & 4 & $2-3$ & 2 & $\begin{array}{l}\text { Deschampsia flexuosa (L.) } \\
\text { Trin. }\end{array}$ & 0.50 & I \\
\hline HT & $\mathrm{x}$ & 5 & $\mathrm{x}$ & 6 & 8 & 4 & 6 & $\mathrm{n}$ & 9 & $3-4$ & 3 & Dactylis glomerata L. s. str. & 0.50 & II \\
\hline HT & $\mathrm{x}$ & 6 & $\mathrm{x}$ & 6 & 6 & 4 & 6 & $\mathrm{n}$ & 9 & $2-4$ & 2 & Festuca pratensis Huds. s. l. & 0.50 & I \\
\hline HT & $\mathrm{x}$ & 5 & $\mathrm{x}$ & $\mathrm{x}$ & 9 & 7 & 6 & $\mathrm{n}$ & 7 & 5 & 3 & Festuca rubra L. & 1.29 & III \\
\hline HT & 5 & 4 & $\mathrm{x}$ & 4 & 6 & 4 & 4 & $\mathrm{n}$ & 6 & $2-3$ & 2 & Holcus lanatus $L$. & 8.26 & IV \\
\hline $\mathrm{H}$ & 5 & 3 & 7 & 2 & & & & $\mathrm{n}$ & 6 & & & Phleum montanum K. Koch & 0.50 & I \\
\hline HT & $\mathrm{x}$ & 5 & $\mathrm{x}$ & 6 & 8 & 6 & 6 & $\mathrm{n}$ & 9 & & & Phleum pratense L. s. str. & 0.50 & III \\
\hline HT & $\mathrm{x}$ & 5 & $\mathrm{x}$ & $\mathrm{x}$ & 9 & 8 & 8 & $\mathrm{n}$ & 8 & $3-5$ & 3 & Poa pratensis L. s. str. & 0.50 & II \\
\hline \multirow[t]{2}{*}{ HT } & $\mathrm{x}$ & $\mathrm{x}$ & $\mathrm{x}$ & 6 & 7 & 5 & 4 & $\mathrm{n}$ & 7 & 3 & 2 & $\begin{array}{l}\text { Trisetum flavescens (L.) P. } \\
\text { Beauv. }\end{array}$ & 0.50 & I \\
\hline & & & & & & & & & & & & CYPERACEAE & 1.5 & \\
\hline \multirow[t]{2}{*}{ HT } & 4 & $\mathrm{x}$ & 4 & 4 & 4 & 5 & 5 & $\mathrm{n}$ & 4 & $2-3$ & 1 & Carex pallescens $L$. & 0.50 & II \\
\hline & & & & & & & & & & & & Luzula luzulina & 0.50 & I \\
\hline HT & $\mathrm{x}$ & $\mathrm{x}$ & 3 & 4 & 4 & 5 & 4 & $\mathrm{n}$ & 4 & $2-3$ & 2 & $\begin{array}{l}\text { Luzula luzuloides (Lam.) } \\
\text { Dandy \& Wilm. }\end{array}$ & 0.50 & II \\
\hline
\end{tabular}




\begin{tabular}{|c|c|c|c|c|c|c|c|c|c|c|c|c|c|c|}
\hline & & & & & & & & & & & & FABACEAE & 18.77 & \\
\hline HT & $\mathrm{x}$ & 4 & 8 & 3 & 4 & 4 & 4 & $\mathrm{n}$ & 6 & $2-3$ & 1 & Anthyllis vulneraria L. s. l. & 0.50 & II \\
\hline $\mathrm{H}$ & 5 & 4 & 9 & 3 & 3 & 7 & & $\mathrm{n}$ & 1 & $2-4$ & 2 & Coronilla varia $L$. & 0.50 & II \\
\hline HT & $\mathrm{x}$ & 4 & 7 & 4 & 6 & 4 & 4 & $\mathrm{n}$ & 7 & $2-4$ & 3 & Lotus corniculatus L. & 1.76 & V \\
\hline $\mathrm{H}$ & 6 & 3 & 9 & 3 & 5 & 2 & 2 & $\mathrm{n}$ & 7 & $2-4$ & 2 & Medicago falcata L. s. str. & 0.50 & I \\
\hline TT & 5 & 4 & $\mathrm{x}$ & 6 & 6 & 4 & 4 & $\mathrm{n}$ & 7 & $3-4$ & 2 & Trifolium campestre Schreb. & 0.50 & II \\
\hline HT & 5 & 6 & 7 & 6 & 7 & 4 & 4 & $\mathrm{n}$ & 8 & $3-4$ & 2 & Trifolium hybridum L. & 0.50 & III \\
\hline \multirow[t]{2}{*}{ HT } & $\mathrm{x}$ & 3 & 8 & 2 & 5 & 4 & 4 & $\mathrm{n}$ & 6 & $2-3$ & 1 & Trifolium montanum $L$. & 3.71 & II \\
\hline & & & & & & & & & & & & Trifolium panonicum L. & 0.50 & III \\
\hline HT & $\mathrm{x}$ & $\mathrm{x}$ & $\mathrm{x}$ & 6 & 7 & 4 & 4 & $\mathrm{n}$ & 8 & $3-4$ & 2 & Trifolium pratense $L$. & 7.52 & $\mathrm{~V}$ \\
\hline ChRs & $\mathrm{x}$ & $\mathrm{x}$ & $\mathrm{x}$ & 6 & 8 & 8 & 8 & $\mathrm{n}$ & 8 & $3-5$ & 3 & Trifolium repens $L$. & 2.28 & $\mathrm{~V}$ \\
\hline \multirow[t]{2}{*}{$\mathrm{HA}$} & $\mathrm{x}$ & 5 & $\mathrm{x}$ & 6 & 6 & 1 & 2 & $\mathrm{n}$ & 6 & $3-4$ & 2 & Vicia cracca L. s. str. & 0.50 & II \\
\hline & & & & & & & & & & & & $\begin{array}{l}\text { OTHER FAMILIES BOTANICAL } \\
(A F B)\end{array}$ & 58.83 & \\
\hline HT & 5 & 4 & 7 & 3 & 7 & 4 & 5 & $\mathrm{n}$ & 4 & & & $\begin{array}{l}\text { Achillea distans Waldst. \& Kit. } \\
\text { ex Willd. }\end{array}$ & 1.50 & II \\
\hline ChRs & $\mathrm{x}$ & 4 & $\mathrm{x}$ & 5 & 7 & 4 & 5 & $\mathrm{n}$ & 6 & $2-4$ & 3 & Achillea millefolium $L$. & 1.38 & IV \\
\hline HRs & 6 & 4 & 8 & 4 & 3 & 4 & 3 & $\mathrm{n}$ & 3 & $2-3$ & 2 & Agrimonia eupatoria L. & 0.50 & I \\
\hline \multirow[t]{2}{*}{ GRs } & $\mathrm{x}$ & $\mathrm{x}$ & 5 & $\mathrm{x}$ & 5 & 6 & 7 & $\mathrm{n}$ & 1 & $2-3$ & 2 & Anemone nemorosa $L$. & 0.50 & I \\
\hline & & & & & & & & & & & & Aposeris foetida & 0.50 & I \\
\hline HRs & 4 & 5 & 3 & 2 & 4 & 4 & 4 & 4 & 4 & $2-3$ & 1 & Arnica montana L. & 0.50 & I \\
\hline HRs & 6 & 4 & $\mathrm{x}$ & 4 & 4 & 2 & 4 & $\mathrm{n}$ & 4 & $2-3$ & 1 & Betonica officinalis $L$. & 0.80 & III \\
\hline ChLS & 4 & 4 & 2 & 4 & 1 & 8 & 9 & $\mathrm{n}$ & 3 & 2 & 1 & $\begin{array}{l}\text { Bruckenthalia spiculifolia } \\
\text { (Salisb.) Rchb. }\end{array}$ & 0.50 & I \\
\hline \multirow[t]{2}{*}{ ChLT } & $\mathrm{x}$ & $\mathrm{x}$ & 1 & 1 & 2 & 5 & 4 & $\mathrm{n}$ & 3 & $2-3$ & 1 & Calluna vulgaris (L.) Hull & 0.50 & I \\
\hline & & & & & & & & & & & & Campanula abietina Griseb. & 0.50 & III \\
\hline \multirow[t]{2}{*}{ HRs } & 5 & 5 & 7 & 4 & 5 & 2 & 2 & $\mathrm{n}$ & 4 & $2-3$ & 2 & Campanula patula L. & 0.50 & IV \\
\hline & & & & & & & & & & & & Campanula persicifolia & 0.50 & III \\
\hline $\mathrm{H}$ & $\mathrm{x}$ & 4 & $\mathrm{x}$ & 2 & 3 & 8 & 7 & $\mathrm{n}$ & 3 & $2-3$ & 1 & Carlina acaulis $L$. & 0.50 & II \\
\hline HRs & 4 & 5 & $\mathrm{x}$ & 6 & 6 & 6 & 6 & $\mathrm{n}$ & 6 & $3-4$ & 1 & Carum carvi $L$. & 0.95 & III \\
\hline HRs & $\mathrm{x}$ & 5 & 5 & 4 & 5 & 2 & 3 & $\mathrm{n}$ & 4 & $2-3$ & 2 & $\begin{array}{l}\text { Centaurea pseudophrygia C. } \\
\text { A. Mey. }\end{array}$ & 3.65 & IV \\
\hline HT & $\mathrm{x}$ & 5 & $\mathrm{x}$ & 5 & 8 & 4 & 4 & $\mathrm{n}$ & 4 & $3-5$ & 3 & Cerastium holosteoides Fr. & 0.50 & III \\
\hline HRs & 5 & 5 & 6 & 5 & 6 & 2 & 2 & $\mathrm{n}$ & 4 & $3-4$ & 3 & Crepis biennis $L$. & 0.50 & III \\
\hline HRs & 6 & 4 & $\mathrm{x}$ & 4 & 6 & 3 & 4 & $\mathrm{n}$ & 5 & $3-5$ & 3 & Daucus carota $L$. & 0.50 & III \\
\hline HRs & 5 & 4 & 7 & 2 & 3 & 4 & 4 & $\mathrm{n}$ & 4 & $2-3$ & 2 & Dianthus carthusianorum L. & 0.50 & I \\
\hline TT & 7 & $\mathrm{x}$ & $\mathrm{x}$ & 4 & 2 & 3 & 2 & $\mathrm{n}$ & 4 & $3-5$ & 3 & Echium vulgare L. & 0.50 & $\mathrm{I}$ \\
\hline TRs & $\mathrm{x}$ & 5 & $\mathrm{x}$ & 3 & 5 & 6 & 5 & $\mathrm{n}$ & 3 & $2-4$ & 1 & $\begin{array}{l}\text { Euphrasia rostkoviana } \\
\text { (Hayne) Towns. }\end{array}$ & 0.50 & III \\
\hline HA & $\mathrm{x}$ & 5 & 7 & $\mathrm{x}$ & 7 & 3 & 3 & $\mathrm{n}$ & 4 & $2-5$ & 3 & Galium album Mill. & 1.06 & III \\
\hline $\mathrm{HT}$ & 5 & 4 & 7 & 3 & 5 & 4 & 4 & $\mathrm{n}$ & 5 & $2-3$ & 2 & Galium verum L. s. str. & 0.91 & II \\
\hline ChLT & 5 & $\mathrm{x}$ & 4 & 2 & 3 & 5 & 4 & $\mathrm{n}$ & 3 & $2-3$ & 1 & Genista tinctoria $L$. & 0.50 & I \\
\hline HT & $\mathrm{x}$ & 5 & 2 & 2 & 3 & 2 & 2 & 4 & 4 & 3 & 1 & Gentiana asclepiadea L. & 0.50 & I \\
\hline HRs & $\mathrm{x}$ & 6 & 3 & 2 & 3 & 3 & 3 & $\mathrm{n}$ & 4 & $2-3$ & 1 & $\begin{array}{l}\text { Hypericum maculatum Crantz } \\
\text { s. str. }\end{array}$ & 0.50 & II \\
\hline HRs & $\mathrm{x}$ & 4 & $\mathrm{x}$ & $\mathrm{x}$ & 3 & 4 & 4 & $\mathrm{n}$ & 1 & $2-6$ & 3 & Hypericum perforatum $L$. & 0.50 & I \\
\hline
\end{tabular}




\begin{tabular}{|c|c|c|c|c|c|c|c|c|c|c|c|c|c|c|}
\hline HRs & 5 & 6 & 6 & 6 & 4 & 2 & 2 & $\mathrm{n}$ & 4 & $2-3$ & 1 & Knautia dipsacifolia Kreutzer & 0.50 & I \\
\hline & & & & & & & & & & & & Laserpitium latifolium $L$. & 0.50 & II \\
\hline HR & $\mathrm{x}$ & 5 & $\mathrm{x}$ & 5 & 7 & 7 & 7 & $\mathrm{n}$ & 5 & $3-4$ & 3 & Leontodon autumnalis $L$. & 0.50 & II \\
\hline \multirow[t]{3}{*}{$\mathrm{H}$} & $\mathrm{x}$ & 4 & $\mathrm{x}$ & 3 & 6 & 3 & 4 & $\mathrm{n}$ & 5 & $3-4$ & 2 & $\begin{array}{l}\text { Leucanthemum vulgare Lam. } \\
\text { s. str. }\end{array}$ & 2.48 & IV \\
\hline & & & & & & & & & & & & $\begin{array}{l}\text { Myosotis sylvatica Ehrh. ex } \\
\text { Hoffm. }\end{array}$ & 0.50 & I \\
\hline & & & & & & & & & & & & Origanum vulgare & 0.50 & I \\
\hline HRs & $\mathrm{x}$ & 6 & 7 & 7 & 5 & 3 & 2 & $\mathrm{n}$ & 5 & $2-3$ & 1 & Pimpinella major (L.) Huds. & 0.50 & III \\
\hline HR & $\mathrm{x}$ & $\mathrm{x}$ & $\mathrm{x}$ & $\mathrm{x}$ & 7 & 6 & 6 & $\mathrm{n}$ & 6 & $2-4$ & 3 & Plantago lanceolata $L$. & 0.50 & IV \\
\hline $\mathrm{HR}$ & $\mathrm{x}$ & 4 & 8 & 3 & 4 & 8 & 8 & $\mathrm{n}$ & 5 & $2-4$ & 2 & Plantago media L. & 0.50 & III \\
\hline HT & $\mathrm{x}$ & 5 & 3 & 2 & 4 & 4 & 4 & $\mathrm{n}$ & 4 & $2-3$ & 1 & Polygala vulgaris L. s. l. & 0.50 & I \\
\hline HT & $\mathrm{x}$ & $\mathrm{x}$ & $\mathrm{x}$ & 2 & 3 & 4 & 5 & $\mathrm{n}$ & 5 & $2-3$ & 1 & Potentilla erecta (L.) Raeusch. & 0.50 & $\mathrm{I}$ \\
\hline HS & $\mathrm{x}$ & $\mathrm{x}$ & 4 & $\mathrm{x}$ & 9 & 8 & 8 & $\mathrm{n}$ & 4 & $3-4$ & 2 & Prunella vulgaris $L$. & 0.50 & IV \\
\hline \multirow[t]{2}{*}{ HRs } & $\mathrm{x}$ & $\mathrm{x}$ & $\mathrm{x}$ & $\mathrm{x}$ & 6 & 5 & 6 & $\mathrm{n}$ & 4 & $3-4$ & 2 & Ranunculus acris $L$. & 0.50 & III \\
\hline & & & & & & & & & & & & Rhinanthus glaber $L$. & 7.67 & $\mathrm{I}$ \\
\hline TRs & $\mathrm{x}$ & $\mathrm{x}$ & $\mathrm{x}$ & 3 & 5 & 8 & 3 & $\mathrm{n}$ & 3 & $2-3$ & 1 & Rhinanthus minor $L$. & 9.45 & II \\
\hline \multirow[t]{2}{*}{ HRs } & $\mathrm{x}$ & $\mathrm{x}$ & $\mathrm{x}$ & $\mathrm{x}$ & 6 & 4 & 2 & $\mathrm{n}$ & 4 & $3-4$ & 2 & Rumex acetosa $L$. & 0.50 & IV \\
\hline & & & & & & & & & & & & Salvia officinalis & 0.50 & IV \\
\hline HRs & 5 & 4 & 8 & 2 & 5 & 3 & 4 & $\mathrm{n}$ & 4 & $2-3$ & 1 & Scabiosa columbaria L. & 0.74 & IV \\
\hline HRs & 5 & 3 & 7 & 3 & 4 & 2 & 2 & $\mathrm{n}$ & 4 & $2-3$ & 2 & Silene nutans $L$. & 0.50 & I \\
\hline HT & $\mathrm{x}$ & $\mathrm{x}$ & 7 & 6 & 4 & 2 & 2 & $\mathrm{n}$ & 4 & $3-4$ & 2 & $\begin{array}{l}\text { Silene vulgaris (Moench) } \\
\text { Garcke s. l. }\end{array}$ & 0.50 & I \\
\hline HS & $\mathrm{x}$ & 4 & 4 & $\mathrm{x}$ & 4 & 5 & 5 & $\mathrm{n}$ & 1 & $2-4$ & 2 & Stellaria graminea $L$. & 0.75 & V \\
\hline HR & $\mathrm{x}$ & 5 & $\mathrm{x}$ & 6 & 8 & 7 & 7 & $\mathrm{n}$ & 7 & $3-5$ & 3 & $\begin{array}{l}\text { Taraxacum officinale Weber } \\
\text { s. l. }\end{array}$ & 0.50 & I \\
\hline ChLS & $\mathrm{x}$ & 2 & 5 & 6 & 4 & 6 & 6 & $\mathrm{n}$ & 3 & $2-3$ & 2 & Thymus serpyllum L. & 0.50 & II \\
\hline HRs & 5 & 4 & 7 & 6 & 6 & 2 & 2 & $\mathrm{n}$ & 5 & $3-4$ & 2 & Tragopogon pratensis L. s. l. & 0.50 & $\mathrm{I}$ \\
\hline ChLS & $\mathrm{x}$ & $\mathrm{x}$ & 2 & 3 & 1 & 8 & 9 & $\mathrm{n}$ & 3 & $2-3$ & 2 & Vaccinium myrtillus L. & 0.50 & I \\
\hline ChLS & $\mathrm{x}$ & 4 & 2 & 1 & 1 & 9 & 9 & $\mathrm{n}$ & 3 & $2-3$ & 1 & Vaccinium vitis_idaea $L$. & 0.50 & I \\
\hline ChRs & $\mathrm{x}$ & 4 & $\mathrm{x}$ & 6 & 7 & 6 & 6 & $\mathrm{n}$ & 4 & $2-4$ & 2 & Veronica chamaedrys L. s. str. & 0.50 & III \\
\hline ChRs & $\mathrm{x}$ & 4 & 2 & 6 & 4 & 4 & 4 & $\mathrm{n}$ & 4 & $2-3$ & 1 & Veronica officinalis $L$. & 0.50 & I \\
\hline HT & 5 & $\mathrm{x}$ & $\mathrm{x}$ & 6 & 4 & 2 & 2 & $\mathrm{n}$ & 4 & $2-3$ & 1 & Viola canina L. s. str. & 0.50 & I \\
\hline HT & 5 & $\mathrm{x}$ & $\mathrm{x}$ & 6 & 5 & 4 & 4 & $\mathrm{n}$ & 4 & $3-5$ & 2 & Viola tricolor $L$. & 0.50 & III \\
\hline
\end{tabular}

are Fabaceae-symmetric (obliquity $=0.98$ ) and platicurtic distribution (vaulting $=-1.10$ ).

From Fabaceae species of the best participation has Trifolium pratense $(7.52 \%)$ followed by the species Trifolium montanum (3.71\%), other species with lower Adm. Lotus corniculatus, Trifolium repens and Trifolium pratense species are enlightening. Are not accompanying species, and some species are random (Trifolium hybridum and Trifolium panonicum). Plants from other botanical families (AFB) are present in phytocoenosis type Agrostis capillaris on average by $58.83 \%$, reaching a minimum of $9 \%$ and a maximum of $52.5 \%$. Obtained data on the participation of plants from other botanical families (are symmetrical, positive (obliquity $=0.20$ ) and platykurtic vaulting $=-0.164$ ). Some species in this group have a significant Adm such as: Rhinanthus minor $9.45 \%$ to $7.67 \%$ Rhinanthus glaber, Centaurea pseudophrygia $3.65 \%$, Leucanthemum vulgare $2.48 \%$ and Galium album with $1.06 \%$. Some species have a lower participation, on average, from 0.74 to $0.95 \%$, as species: Scabiosa columbaria, Stellaria 
Tab. 2 Ecological and agronomical spectrum of Agrostis capillaries grassland type

\begin{tabular}{|c|c|c|c|c|c|c|c|c|c|c|c|}
\hline \multirow{2}{*}{$\begin{array}{c}\text { Eclg. in } \\
\text { dexes } \\
1\end{array}$} & \multicolumn{10}{|c|}{ Ecological spectrum } & \multirow[t]{2}{*}{ VIMnp } \\
\hline & 2 & 3 & 4 & 5 & 6 & 7 & 8 & 9 & $\mathrm{x}$ & VIMp & \\
\hline Unp & 0.0 & 1.0 & 5.0 & 26.0 & 19.0 & 5.0 & 0.0 & 0.0 & 0.0 & 23 & 4.4 \\
\hline Up & 0.0 & 0.5 & 6.0 & 33.6 & 15.5 & 2.5 & 0.0 & 0.0 & 0.0 & 54.69 & 4.2 \\
\hline Rnp & 1.0 & 6.0 & 4.0 & 4.0 & 5.0 & 2.0 & 14.0 & 6.0 & 2.0 & 35.00 & 5.5 \\
\hline $\mathbf{R p}$ & 0.5 & 3.0 & 2.0 & 2.3 & 8.2 & 1.0 & 14.7 & 6.5 & 1.0 & 73.64 & 5.9 \\
\hline Nnp & 2.0 & 11.0 & 13.0 & 14.0 & 5.0 & 22.0 & 1.0 & 0.0 & 0.0 & 11.00 & 4.2 \\
\hline $\mathrm{Np}$ & 1.0 & 9.0 & 18.8 & 48.2 & 3.4 & 22.5 & 0.5 & 0.0 & 0.0 & 9.34 & 4.1 \\
\hline \multirow{2}{*}{$\begin{array}{c}\text { Agron. } \\
\text { indexes } \\
1\end{array}$} & \multicolumn{10}{|c|}{ Agronomic spectrum } & VIMnp \\
\hline & 2 & 3 & 4 & 5 & 6 & 7 & 8 & 9 & $x$ & VIMp & \\
\hline Cnp & 3.0 & 3.0 & 12.0 & 16.0 & 11.0 & 13.0 & 11.0 & 6.0 & 3.0 & 0.00 & 5.1 \\
\hline $\mathrm{Cp}$ & 1.5 & 1.5 & 12.5 & 8.6 & 21.5 & 41.2 & 18.2 & 5.1 & 2.3 & 0.00 & 5.5 \\
\hline Pnp & 1.0 & 11.0 & 8.0 & 25.0 & 9.0 & 8.0 & 6.0 & 8.0 & 1.0 & 0.00 & 4.6 \\
\hline Pp & 0.5 & 9.0 & 8.8 & 34.0 & 30.2 & 9.0 & 5.1 & 14.7 & 0.5 & 0.00 & 4.9 \\
\hline Snp & 0.0 & 13.0 & 6.0 & 25.0 & 8.0 & 10.0 & 7.0 & 5.0 & 3.0 & 0.00 & 4.7 \\
\hline Sp & 0.0 & 6.5 & 15.7 & 34.7 & 31.6 & 10.7 & 6.8 & 4.3 & 1.5 & 0.00 & 4.6 \\
\hline VFnp & 0.0 & 1.0 & 0.0 & 4.0 & 0.0 & 0.0 & 0.0 & 0.0 & 0.0 & 0 & 3.6 \\
\hline VFp & 0.0 & 0.5 & 0.0 & 4.3 & 0.0 & 0.0 & 0.0 & 0.0 & 0.0 & 0 & 3.8 \\
\hline & & & & & & & & & & & \\
\hline $\mathbf{U}$ & & & C & & mow & & VF & & fodc & ralue & \\
\hline $\mathbf{R}$ & soil & ion & $\mathbf{P}$ & & grazi & & np & unweig & $\begin{array}{l}\text { (dep } \\
\text { of } s\end{array}$ & $\begin{array}{l}\text { ng on th } \\
\text { ies) }\end{array}$ & number \\
\hline $\mathbf{N}$ & & & $\mathbf{S}$ & & crush & & $\mathbf{p}$ & & $\begin{array}{r}d \text { dep } \\
\text { co }\end{array}$ & $\begin{array}{l}\text { ling on } \\
\text { ge) }\end{array}$ & ecies \\
\hline
\end{tabular}

graminea, Galium verum and Carum carvi. Many species have a low participation, averaging $0.5 \%$.

Only one edifying species is present of this type of grassland from AFB group plants which is Stellaria graminea $(\mathrm{K}=\mathrm{V})$. Common species $(\mathrm{K}$ = IV) are: Achillea millefolium, Campanula patula, Centaurea pseudophrygia, Leucanthemum vulgare, Plantago lanceolata, Prunella vulgaris, Rumex acetosa and Scabiosa columbaria.

Among the indicator species ( $\mathrm{K}=\mathrm{III})$ encountered in this type of meadow include: Betonica officinalis, Campanula abietina, Campanula persicifolia, Carum carvi, Cerastium holosteoides, Crepis biennis, Daucus carota, Euphrasia rostkoviana, Galium album, Pimpinella major, Plantago media, Ranunculus acris, Veronica chamaedrys and Viola tricolor. There are 7 species accompanying and 26 species considered accidental.

From ecological point of view (ecological spectrum) phytocoenosis has a meso-xerophylous
$(\mathrm{Up}=4.2)$, moderate acidophilous $(\mathrm{Rp}=5.9)$ and oligomezotrophic $(\mathrm{Np}=4.1)$ characters.

The agronomical point of view (agronomical spectrum) phytocoenosis has medium character tolerant mowing ( $\mathrm{Cp}=5.5)$, is moderately tolerant grazing $(\mathrm{Pp}=4.9)$ and moderately tolerant crushed $(\mathrm{Sp}=4.6)$. The interpretation of agronomic factors, we can conclude that the grasslands are used in a semi-extensive mode, the plants are trampled frequently in grazing period (Tab. 2).

Thus, the feed value of Agrostis capillaries grassland type is 3.8 (VFP = 3.8), so meadow covered by Class IV category of mediocre pasture and supports from 0.41 to $0.60 \mathrm{LU} / \mathrm{ha}$.

The agronomical point of view in phytocoenosis, type has not the toxic species only a damaging species of animal products. Phytocoenosis presents coverage of $4.3 \%$ with poor forage species (ballast). Average or good forage species missing from the grassy coverage. 


\section{CONCLUSIONS}

Interpretation of agronomic factors we can conclude that the grasslands are used in a semiextensive mode, these surfaces being under-exploited.

Thus, the feed value of Agrostis capillaries grassland type is 3.8 (VFP $=3.8$ ), so meadow covered by Class IV category of mediocre pasture and supports from 0.41 to $0.60 \mathrm{LU} / \mathrm{ha}$.

The average data from 29 plots of results that Agrostis capillaries it has a range between 17.50 and $27.50 \%$. The Fabaceae family have coverage between 5 and $19 \%$.

The floristic composition of the Agrostis $\mathrm{Ca}$ pillaries grasslands from the studied area is influenced by the local conditions and the intensity of the exploitation, these surfaces being under-exploited.

We also are militating for a properly grazing system and minimal maintenance works.

\section{REFERENCES}

1. Cristea V, Gafta D, Pedrotti F (2004). Fitosociologie, Publisher Presa Universitară Clujeană (Chapter IV).

2. Dierschke H, Briemle G, Kratochwil A (2002). Kulturgrasland: Wiesen, Weiden und verwandte Staudenfluren. E. Ulmer

3. Gârda N (2010). Studiul unor elemente de landșaft montan (cu privire specială asupra ecosistemelor de pajiști din comuna Gârda de Sus, Munții Apuseni), Teză de doctorat USAMV Cluj- Napoca.

4. Păcurar F, Rotar I, (2014 a). Metode de studiu și interpretare a vegetației pajiștilor, Ed. Risoprint, Cluj-Napoca.

5. Țucra I, Kovacs AJ, Roșu C, Ciubotariu C, Chifu T, Neacșu M, Bărbulescu C, Cardaşol V, Popovici D, Simtea N, Motcă G, Dragu I, Spirescu M (1987). Principalele tipuri de pajişti din R. S. România, Ed. Poligrafică "Bucureștii Noi".

6. ***, (http://statisticasociala.tripod.com/parametri.htm.

Annex 1. Statistic processing of data regarding stand conditions, species composition of Agrostis capillaris L. type and the species' frequence constancy in relevees

\begin{tabular}{lccccccccccc}
\hline Species & Average & Stdev & CV & Median & Mode & Skew & Kurt & Min & Max & Count & K \\
\hline POACEAE & & & & & & & & & & & \\
\hline Agrostis capillaris L. & 23.71 & 4.94 & 0.21 & 27.50 & 27.50 & -0.53 & -1.86 & 17.50 & 27.50 & 29.00 & V \\
\hline Anthoxanthum odoratum L. s. str. & 2.73 & 2.73 & 1.00 & 2.75 & 2.75 & 2.57 & 7.13 & 0.50 & 11.50 & 27.00 & V \\
\hline Briza media L. & 0.50 & 0.00 & 0.00 & 0.50 & 0.50 & 0.00 & 0.00 & 0.50 & 0.50 & 18.00 & IV \\
\hline Bromus inermis Leyss. & 0.50 & 0.00 & 0.00 & 0.50 & 0.50 & 0.00 & 0.00 & 0.50 & 0.50 & 3.00 & I \\
\hline Cynosurus cristatus L. & 1.53 & 1.15 & 0.75 & 0.50 & 0.50 & 0.18 & -2.16 & 0.50 & 2.75 & 24.00 & V \\
\hline Deschampsia flexuosa (L.) Trin. & 0.50 & 0.00 & 0.00 & 0.50 & 0.50 & 0.00 & 0.00 & 0.50 & 0.50 & 5.00 & I \\
\hline Dactylis glomerata L. s. str. & 0.50 & 0.00 & 0.00 & 0.50 & 0.50 & 0.00 & 0.00 & 0.50 & 0.50 & 11.00 & II \\
\hline Festuca pratensis Huds. s. l. & 0.50 & 0.00 & 0.00 & 0.50 & 0.50 & 0.00 & 0.00 & 0.50 & 0.50 & 2.00 & I \\
\hline Festuca rubra L. & 1.29 & 1.77 & 1.37 & 0.50 & 0.50 & 1.87 & 1.67 & 0.50 & 5.00 & 17.00 & III \\
\hline Holcus lanatus L. & 8.26 & 5.81 & 0.70 & 11.25 & 11.25 & -0.20 & -1.13 & 0.50 & 17.50 & 18.00 & IV \\
\hline Phleum montanum K. Koch & 0.50 & 0.00 & 0.00 & 0.50 & 0.50 & 0.00 & 0.00 & 0.50 & 0.50 & 5.00 & I \\
\hline Phleum pratense L. s. str. & 0.50 & 0.00 & 0.00 & 0.50 & 0.50 & 0.00 & 0.00 & 0.50 & 0.50 & 12.00 & III \\
\hline Poa pratensis L. s. str. & 0.50 & 0.00 & 0.00 & 0.50 & 0.50 & 0.00 & 0.00 & 0.50 & 0.50 & 7.00 & II \\
\hline Trisetum flavescens (L.) P. Beauv. & 0.50 & 0.00 & 0.00 & 0.50 & 0.50 & 0.00 & 0.00 & 0.50 & 0.50 & 3.00 & I \\
\hline CYPERACEAE & 0.00 & 0.00 & 0.00 & 0.00 & 0.00 & 0.00 & 0.00 & 0.00 & 0.00 & 0.00 & I \\
\hline Carex pallescens L. & 0.50 & 0.00 & 0.00 & 0.50 & 0.50 & 0.00 & 0.00 & 0.50 & 0.50 & 9.00 & II \\
\hline Luzula luzulina & 0.50 & 0.00 & 0.00 & 0.50 & 0.50 & 0.00 & 0.00 & 0.50 & 0.50 & 5.00 & I \\
\hline Luzula luzuloides (Lam.) Dandy \& & 0.50 & 0.00 & 0.00 & 0.50 & 0.50 & 0.00 & 0.00 & 0.50 & 0.50 & 7.00 & II \\
Wilm. & & & & & & & & & & \\
\hline
\end{tabular}




\begin{tabular}{|c|c|c|c|c|c|c|c|c|c|c|c|}
\hline FABACEAE & 0.00 & 0.00 & 0.00 & 0.00 & 0.00 & 0.00 & 0.00 & 0.00 & 0.00 & 0.00 & $\mathrm{I}$ \\
\hline Anthyllis vulneraria L. s. l. & 0.50 & 0.00 & 0.00 & 0.50 & 0.50 & 0.00 & 0.00 & 0.50 & 0.50 & 7.00 & II \\
\hline Coronilla varia $L$. & 0.50 & 0.00 & 0.00 & 0.50 & 0.50 & 0.00 & 0.00 & 0.50 & 0.50 & 10.00 & II \\
\hline Lotus corniculatus L. & 1.76 & 1.73 & 0.98 & 0.50 & 0.50 & 0.98 & -0.51 & 0.50 & 5.00 & 25.00 & $\mathrm{~V}$ \\
\hline Medicago falcata L. s. str. & 0.50 & 0.00 & 0.00 & 0.50 & 0.50 & 0.00 & 0.00 & 0.50 & 0.50 & 4.00 & I \\
\hline Trifolium campestre Schreb. & 0.50 & 0.00 & 0.00 & 0.50 & 0.50 & 0.00 & 0.00 & 0.50 & 0.50 & 8.00 & II \\
\hline Trifolium hybridum $L$. & 0.50 & 0.00 & 0.00 & 0.50 & 0.50 & 0.00 & 0.00 & 0.50 & 0.50 & 12.00 & III \\
\hline Trifolium montanum $L$. & 3.71 & 2.20 & 0.59 & 5.00 & 5.00 & -1.23 & -0.84 & 0.50 & 5.00 & 7.00 & II \\
\hline Trifolium panonicumL. & 0.50 & 0.00 & 0.00 & 0.50 & 0.50 & 0.00 & 0.00 & 0.50 & 0.50 & 12.00 & III \\
\hline Trifolium pratense $L$. & 7.52 & 4.92 & 0.65 & 5.00 & 11.25 & 0.31 & -0.99 & 0.50 & 17.50 & 29.00 & $\mathrm{~V}$ \\
\hline Trifolium repens $L$. & 2.28 & 1.99 & 0.87 & 1.63 & 0.50 & 0.44 & -1.62 & 0.50 & 5.00 & 24.00 & $\mathrm{~V}$ \\
\hline Vicia cracca L. s. str. & 0.50 & 0.00 & 0.00 & 0.50 & 0.50 & 0.00 & 0.00 & 0.50 & 0.50 & 10.00 & II \\
\hline $\begin{array}{l}\text { OTHER FAMILIES BOTANICAL } \\
\text { (AFB) }\end{array}$ & 0.00 & 0.00 & 0.00 & 0.00 & 0.00 & 0.00 & 0.00 & 0.00 & 0.00 & 0.00 & I \\
\hline $\begin{array}{l}\text { Achillea distans Waldst. \& Kit. ex } \\
\text { Willd. }\end{array}$ & 1.50 & 1.19 & 0.79 & 0.50 & 0.50 & 0.27 & -2.57 & 0.50 & 2.75 & 9.00 & II \\
\hline Achillea millefolium $L$. & 1.38 & 1.13 & 0.82 & 0.50 & 0.50 & 0.50 & -1.99 & 0.50 & 2.75 & 18.00 & IV \\
\hline Agrimonia eupatoria L. & 0.50 & 0.00 & 0.00 & 0.50 & 0.50 & 0.00 & 0.00 & 0.50 & 0.50 & 3.00 & I \\
\hline Anemone nemorosa $L$. & 0.50 & 0.00 & 0.00 & 0.50 & 0.50 & 0.00 & 0.00 & 0.50 & 0.50 & 2.00 & $\mathrm{I}$ \\
\hline Aposeris foetida & 0.50 & 0.00 & 0.00 & 0.50 & 0.50 & 0.00 & 0.00 & 0.50 & 0.50 & 2.00 & I \\
\hline Arnica montana L. & 0.50 & 0.00 & 0.00 & 0.50 & 0.50 & 0.00 & 0.00 & 0.50 & 0.50 & 5.00 & I \\
\hline Betonica officinalis $L$. & 0.80 & 0.79 & 0.99 & 0.50 & 0.50 & 2.40 & 4.35 & 0.50 & 2.75 & 15.00 & III \\
\hline $\begin{array}{l}\text { Brachypodium pinnatum (L.) P. } \\
\text { Beauv. }\end{array}$ & 5.00 & 0.00 & 0.00 & 5.00 & 5.00 & 0.00 & 0.00 & 5.00 & 5.00 & 5.00 & I \\
\hline $\begin{array}{l}\text { Bruckenthalia spiculifolia (Salisb.) } \\
\text { Rchb. }\end{array}$ & 0.50 & 0.00 & 0.00 & 0.50 & 0.50 & 0.00 & 0.00 & 0.50 & 0.50 & 5.00 & I \\
\hline Calluna vulgaris (L.) Hull & 0.50 & 0.00 & 0.00 & 0.50 & 0.50 & 0.00 & 0.00 & 0.50 & 0.50 & 5.00 & $\mathrm{I}$ \\
\hline Campanula abietina Griseb. & 0.50 & 0.00 & 0.00 & 0.50 & 0.50 & 0.00 & 0.00 & 0.50 & 0.50 & 12.00 & III \\
\hline Campanula patula $L$. & 0.50 & 0.00 & 0.00 & 0.50 & 0.50 & 0.00 & 0.00 & 0.50 & 0.50 & 20.00 & IV \\
\hline Campanula persicifolia & 0.50 & 0.00 & 0.00 & 0.50 & 0.50 & 0.00 & 0.00 & 0.50 & 0.50 & 17.00 & III \\
\hline Carlina acaulis $L$. & 0.50 & 0.00 & 0.00 & 0.50 & 0.50 & 0.00 & 0.00 & 0.50 & 0.50 & 11.00 & II \\
\hline Carum carvi L. & 0.95 & 0.93 & 0.98 & 0.50 & 0.50 & 1.67 & 0.90 & 0.50 & 2.75 & 15.00 & III \\
\hline Centaurea pseudophrygia C. A. Mey. & 3.65 & 1.53 & 0.42 & 3.88 & 5.00 & -0.71 & -0.45 & 0.50 & 5.00 & 20.00 & IV \\
\hline Cerastium holosteoides Fr. & 0.50 & 0.00 & 0.00 & 0.50 & 0.50 & 0.00 & 0.00 & 0.50 & 0.50 & 13.00 & III \\
\hline Crepis biennis $L$. & 0.50 & 0.00 & 0.00 & 0.50 & 0.50 & 0.00 & 0.00 & 0.50 & 0.50 & 13.00 & III \\
\hline Daucus carota $L$. & 0.50 & 0.00 & 0.00 & 0.50 & 0.50 & 0.00 & 0.00 & 0.50 & 0.50 & 13.00 & III \\
\hline Dianthus carthusianorum $L$. & 0.50 & 0.00 & 0.00 & 0.50 & 0.50 & 0.00 & 0.00 & 0.50 & 0.50 & 3.00 & $\mathrm{I}$ \\
\hline Echium vulgare $L$. & 0.50 & 0.00 & 0.00 & 0.50 & 0.50 & 0.00 & 0.00 & 0.50 & 0.50 & 5.00 & $\mathrm{I}$ \\
\hline $\begin{array}{l}\text { Euphrasia rostkoviana (Hayne) } \\
\text { Towns. }\end{array}$ & 0.50 & 0.00 & 0.00 & 0.50 & 0.50 & 0.00 & 0.00 & 0.50 & 0.50 & 13.00 & III \\
\hline Galium album Mill. & 1.06 & 1.54 & 1.45 & 0.50 & 0.50 & 2.51 & 4.90 & 0.50 & 5.00 & 16.00 & III \\
\hline Galium verum L. s. str. & 0.91 & 0.91 & 1.00 & 0.50 & 0.50 & 1.92 & 2.04 & 0.50 & 2.75 & 11.00 & II \\
\hline Genista tinctoria $L$. & 0.50 & 0.00 & 0.00 & 0.50 & 0.50 & 0.00 & 0.00 & 0.50 & 0.50 & 3.00 & $\mathrm{I}$ \\
\hline Gentiana asclepiadea $L$. & 0.50 & 0.00 & 0.00 & 0.50 & 0.50 & 0.00 & 0.00 & 0.50 & 0.50 & 5.00 & $\mathrm{I}$ \\
\hline Hypericum maculatum Crantz s. str. & 0.50 & 0.00 & 0.00 & 0.50 & 0.50 & 0.00 & 0.00 & 0.50 & 0.50 & 10.00 & II \\
\hline Hypericum perforatum $L$. & 0.50 & 0.00 & 0.00 & 0.50 & 0.50 & 0.00 & 0.00 & 0.50 & 0.50 & 3.00 & I \\
\hline
\end{tabular}




\begin{tabular}{|c|c|c|c|c|c|c|c|c|c|c|c|}
\hline Knautia dipsacifolia Kreutzer & 0.50 & 0.00 & 0.00 & 0.50 & 0.50 & 0.00 & 0.00 & 0.50 & 0.50 & 2.00 & $\mathrm{I}$ \\
\hline Laserpitium latifolium L. & 0.50 & 0.00 & 0.00 & 0.50 & 0.50 & 0.00 & 0.00 & 0.50 & 0.50 & 9.00 & II \\
\hline Leontodon autumnalis $L$. & 0.50 & 0.00 & 0.00 & 0.50 & 0.50 & 0.00 & 0.00 & 0.50 & 0.50 & 10.00 & II \\
\hline Leucanthemum vulgare Lam. s. str. & 2.48 & 3.19 & 1.29 & 1.63 & 0.50 & 2.29 & 4.82 & 0.50 & 11.25 & 20.00 & IV \\
\hline Myosotis sylvatica Ehrh. ex Hoffm. & 0.50 & 0.00 & 0.00 & 0.50 & 0.50 & 0.00 & 0.00 & 0.50 & 0.50 & 3.00 & $\mathrm{I}$ \\
\hline Origanum vulgare & 0.50 & 0.00 & 0.00 & 0.50 & 0.50 & 0.00 & 0.00 & 0.50 & 0.50 & 6.00 & $\mathrm{I}$ \\
\hline Pimpinella major (L.) Huds. & 0.50 & 0.00 & 0.00 & 0.50 & 0.50 & 0.00 & 0.00 & 0.50 & 0.50 & 15.00 & III \\
\hline Plantago lanceolata L. & 0.50 & 0.00 & 0.00 & 0.50 & 0.50 & 0.00 & 0.00 & 0.50 & 0.50 & 18.00 & IV \\
\hline Plantago media L. & 0.50 & 0.00 & 0.00 & 0.50 & 0.50 & 0.00 & 0.00 & 0.50 & 0.50 & 16.00 & III \\
\hline Polygala vulgaris L. s. l. & 0.50 & 0.00 & 0.00 & 0.50 & 0.50 & 0.00 & 0.00 & 0.50 & 0.50 & 5.00 & $\mathrm{I}$ \\
\hline Potentilla erecta (L.) Raeusch. & 0.50 & 0.00 & 0.00 & 0.50 & 0.50 & 0.00 & 0.00 & 0.50 & 0.50 & 5.00 & $\mathrm{I}$ \\
\hline Prunella vulgaris $L$. & 0.50 & 0.00 & 0.00 & 0.50 & 0.50 & 0.00 & 0.00 & 0.50 & 0.50 & 18.00 & IV \\
\hline Ranunculus acris $L$. & 0.50 & 0.00 & 0.00 & 0.50 & 0.50 & 0.00 & 0.00 & 0.50 & 0.50 & 14.00 & III \\
\hline Rhinanthus glaber L. & 7.67 & 5.55 & 0.72 & 11.25 & 11.25 & -0.97 & -1.88 & 0.50 & 11.25 & 6.00 & $\mathrm{I}$ \\
\hline Rhinanthus minor $L$. & 9.45 & 8.53 & 0.90 & 10.13 & 17.50 & -0.03 & -2.51 & 0.50 & 17.50 & 10.00 & II \\
\hline Rumex acetosa L. & 0.50 & 0.00 & 0.00 & 0.50 & 0.50 & 0.00 & 0.00 & 0.50 & 0.50 & 20.00 & IV \\
\hline Salvia officinalis & 0.50 & 0.00 & 0.00 & 0.50 & 0.50 & 0.00 & 0.00 & 0.50 & 0.50 & 21.00 & IV \\
\hline Scabiosa columbaria L. & 0.74 & 0.71 & 0.96 & 0.50 & 0.50 & 2.80 & 6.51 & 0.50 & 2.75 & 19.00 & IV \\
\hline Silene nutans $L$. & 0.50 & 0.00 & 0.00 & 0.50 & 0.50 & 0.00 & 0.00 & 0.50 & 0.50 & 4.00 & $\mathrm{I}$ \\
\hline Silene vulgaris (Moench) $G$ & 0.50 & 0.00 & 0.00 & 0.50 & 0.50 & 0.00 & 0.00 & 0.50 & 0.50 & 5.00 & $\mathrm{I}$ \\
\hline Stellaria graminea $L$. & 0.75 & 0.72 & 0.96 & 0.50 & 0.50 & 2.62 & 5.27 & 0.50 & 2.75 & 27.00 & $\mathrm{~V}$ \\
\hline Taraxacum officinale Weber s. l. & 0.50 & 0.00 & 0.00 & 0.50 & 0.50 & 0.00 & 0.00 & 0.50 & 0.50 & 2.00 & $\mathrm{I}$ \\
\hline Thymus serpyllum $L$. & 0.50 & 0.00 & 0.00 & 0.50 & 0.50 & 0.00 & 0.00 & 0.50 & 0.50 & 10.00 & II \\
\hline Tragopogon pratensis L. s. l. & 0.50 & 0.00 & 0.00 & 0.50 & 0.50 & 0.00 & 0.00 & 0.50 & 0.50 & 7.00 & $\mathrm{I}$ \\
\hline Vaccinium myrtillus L. & 0.50 & 0.00 & 0.00 & 0.50 & 0.50 & 0.00 & 0.00 & 0.50 & 0.50 & 5.00 & I \\
\hline Vaccinium vitis_idaea $L$. & 0.50 & 0.00 & 0.00 & 0.50 & 0.50 & 0.00 & 0.00 & 0.50 & 0.50 & 5.00 & $\mathrm{I}$ \\
\hline Veronica chamaedrys L. s. str. & 0.50 & 0.00 & 0.00 & 0.50 & 0.50 & 0.00 & 0.00 & 0.50 & 0.50 & 15.00 & III \\
\hline Veronica officinalis $L$. & 0.50 & 0.00 & 0.00 & 0.50 & 0.50 & 0.00 & 0.00 & 0.50 & 0.50 & 5.00 & I \\
\hline Viola canina L. s. str. & 0.50 & 0.00 & 0.00 & 0.50 & 0.50 & 0.00 & 0.00 & 0.50 & 0.50 & 5.00 & I \\
\hline Viola tricolor $L$. & 0.50 & 0.00 & 0.00 & 0.50 & 0.50 & 0.00 & 0.00 & 0.50 & 0.50 & 17.00 & III \\
\hline
\end{tabular}

